\title{
El patrimonio cultural afroargentino: un análisis del programa "ruta del esclavo" UNESCO en Argentina
}

맘 RMA

Afro-argentine cultural heritage: the UNESCO "slave-route" in Argentina

Milena Annecchiarico

Antropología Social

Instituto de Ciencias Antropológicas, Facultad de Filosofía y Letras, Universidad de Buenos Aires, CONICET. E-mail: milargenta@gmail.com

\begin{abstract}
Resumen
Este artículo propone un análisis de la trayectoria del programa UNESCO "Ruta del Esclavo" en Argentina, que promueve la investigación sobre la esclavitud y la trata transatlántica de africanos esclavizados, las formas de resistencia y la valorización de los sitios de memoria y de las manifestaciones culturales de las comunidades afrodescendientes actuales. Se analiza su reciente implementación en Argentina ocurrida a mediados de los años 2000, ubicando el programa en el contexto nacional e internacional de promoción de políticas culturales y de patrimonialización referidas a la memoria de la esclavitud. Destacamos algunos aspectos y problemáticas que nos ayuden a pensar el alcance y los límites de las políticas patrimoniales y culturales desarrolladas en el marco del programa desde una perspectiva local. Consideramos entonces la experiencia de la Capilla de Los Negros de Chascomús, uno de los sitios de memoria de la esclavitud declarados en el marco del programa, poniendo en relieve tensiones y ambivalencias entre los diferentes actores involucrados.
\end{abstract}

Palabras clave: Ruta del Esclavo, Memoria de la Esclavitud, Patrimonio cultural, Afrodescendientes argentinos.

\begin{abstract}
This article analyzes the trajectory of the UNESCO "Slave Route" program in Argentina. This program promotes research on the transatlantic slave trade of Africans, the contemporary forms of resistance and the valorization of memory sites. It also seeks for the recognition of present Afro-descendant communities. The article focuses on the recent implementation of this program in Argentina, placing the memory of slavery it in the context of national and international cultural policies of patrimonialization. From a local perspective, it highlights some aspects and problems that help to understand the scope and limits of cultural and heritage policies. The research on the experience of the Chapel of Los Negros de Chascomús, one of the memory sites of slavery declared within the framework of the program, shows tensions and ambivalences among the different actors involved in this kind of culture policies.
\end{abstract}

Keywords: Unesco Slave Rute; Slave memories; Cultural heritage; Afro-descendants in Argentina.

A mediados de los años 2000, ocurrió un hecho significativo que situó a la República Argentina en el debate internacional sobre la esclavitud y la trata transatlántica de africanos y africanas esclavizados. Junto a Uruguay y Paraguay, Argentina fue incluida en el programa "Ruta del Esclavo" de la Organización de las Naciones Unidas para la Educación, la Ciencia y la Cultura (UNESCO), lanzado en 1994 en Benín e implementado a lo largo de los años en diferentes países del mundo (Guanche, 2011; Pineau, 2011, 2012; UNESCO, 2014). La RdE es un programa transnacional que promueve la investigación sobre la esclavitud y la trata transatlántica de africanos esclavizados, las formas de resistencia y la valorización de los sitios de memoria y de las manifestaciones culturales de las comunidades afrodescendientes, en sus más de veinte años de trayectoria en Europa, África y América (UNESCO, 2014).
El presente artículo aborda la trayectoria en Argentina del programa "Ruta del Esclavo" de UNESCO (en adelante $\mathrm{RdE}$ ) y las activaciones patrimoniales realizadas en ese marco ejemplificadas en el caso de la Capilla de los Negros de Chascomús, de acuerdo a los resultados de mi investigación doctoral (Annecchiarico, 2016). ${ }^{1}$ Para ello, realizamos una selección y revisión critica de la documentación disponible en la web y una etnografía de la emergencia del programa en Argentina, relevando las actividades realizadas en ese marco y los principales actores, destacando límites, alcances y desafíos. Si bien la trayectoria local del programa fue breve, desde finales

\footnotetext{
${ }^{1}$ Este artículo es una versión ampliada y revisada de la ponencia presentada en las VIII Jornadas de investigación en Antropología Cultural Santiago Wallace de la Universidad de Buenos Aires, del 27 al 29 de julio de 2016. Agradezco los comentarios y aportes realizados en esa ocasión.
} 
de los años 2000 hasta el 2014, las acciones realizadas en ese marco constituyen una interesante plataforma de articulación con las nuevas agendas políticas estatales en tema de reconocimiento y visibilización de la población afrodescendiente y, además, representaron una inédita ocasión para la difusión y circulación de los estudios locales sobre estas temáticas. Al mismo tiempo, los proyectos encaminados desde el programa se cruzan con las demandas de los movimientos locales de afrodescendientes, conformando un nuevo escenario en los procesos de patrimonialización de sitios y memorias de la esclavitud en Argentina. ${ }^{2}$

En este ensayo, nos interesa destacar alcances, ambivalencias y desafíos de una política patrimonial afroargentina de acuerdo a algunos interrogantes, cuales: ¿qué contextos favorecieron la conformación de esas políticas? ¿Cuáles fueron las acciones llevadas a cabo y los desafíos que dejaron planteados? ¿Cuáles son los diferentes agentes sociales involucrados? ¿Cuáles narrativas, significados y disputas emergen sobre el pasado, la cultura y la identidad afroargentina?

Considero que el programa RdE de UNESCO es un amplio campo de acción en donde interactúan diferentes dimensiones, culturales, políticas, económicas, sociales, que nos permiten indagar las interconexiones entre políticas nacionales e internacionales sobre cultura, patrimonio y memorias, así como las formas de participación y las demandas de las comunidades afrodescendientes. De acuerdo con una perspectiva atenta a la experiencia y agentividad de los sujetos, considero las disputas y los usos diferenciales de memorias e identidades afro en la arena cultural y política contemporánea.

El artículo comienza con una contextualización de la agenda política nacional respecto de la temática afroargentina luego de 2001 y las discusiones en el contexto internacional de promoción de políticas culturales y políticas de diversidad, en donde la cultura y el patrimonio adquieren nuevos significados. Enfoques recientes que la antropología propone para el estudio del patrimonio cultural, ponen en relieve las articulaciones entre diferentes esferas y lógicas disimiles (culturales, artísticas, mercantilistas, políticas, científicas) y las tensiones al interior de una arena de negociaciones entre agentes sociales desigualmente posicionados, que contribuyen a redefinir identidades, tradiciones, memorias y silencios (Arantes, 1984; Prats, 1997; Rotman, 2001; Pollak, 2006; Crespo et al., 2007; Morel, 2011). A partir de estas consideraciones, presento la estructura de la RdE, los objetivos y las principales etapas de los primeros veinte

\footnotetext{
${ }^{2}$ El término afrodescendiente, que empleo en este artículo, fue lanzado en la Conferencia Mundial contra el Racismo celebrada en Durban en 2001, para nombrar a los descendientes de africanos nacidos fuera del continente africano, principalmente en las Américas, producto del tráfico esclavista. Me refiero por lo tanto a la población afroargentina en estos términos, teniendo en cuenta la heterogeneidad de trayectorias históricas de la población afrodescendiente actual.
}

años de trayectoria, de 1994 a 2014. Abordo entonces la implementación de la RdE en Argentina, relevando los impactos y los desafíos en relación a los debates locales sobre la cuestión afroargentina y a las agendas políticas del estado y de las organizaciones de afrodescendientes. Analizo finalmente un caso significativo de activación patrimonial en el marco de la RdE en Argentina: la Capilla de los Negros de Chascomús. Para ello, destaco los diferentes agentes sociales involucrados en el proceso patrimonial y sus posicionamientos diferenciales: la población afrodescendiente local, el activismo afroargentino, los organismos municipales de cultura, círculos académicos e institucionales. De acuerdo a lecturas recientes de la antropología, los procesos de patrimonialización son arenas de confrontación y disputas a partir de la negociación de "identidades", "tradiciones" y "memorias" (Crespo et al. 2007). Al respecto, destaco algunos efectos, ambivalencias y criticidades a lo largo del proceso, así como las oportunidades movilizadas para la visibilización de la Capilla y los significados emergentes sobre memorias, narrativas e identidades afroargentinas.

El propósito de este trabajo es contribuir a formular nuevas preguntas y afinar visiones que permitan profundizar las interrelaciones entre agendas locales y transnacionales en el campo de las políticas culturales de reconocimiento y de reparación hacia la población afrodescendiente (Mosquera y Barcelos, 2006), articulaciones que visibilizan además las tensiones que intervienen en los procesos patrimoniales de la presencia africana en Argentina.

\section{Afrodescendientes y Estado argentino: el contexto local}

De acuerdo a la literatura especializada, desde las décadas de los 90 se asiste en América Latina a una reconfiguración de la cuestión afrolatinoamericana en diferentes niveles, un período caracterizado por la filosofía política del multiculturalismo globalizado y el consolidarse de redes transnacionales de activismo negro (Hale, 2005; Wade, 2006; Lao-Montes, 2007). Se van perfilando activaciones patrimoniales de memorias de la esclavitud y de prácticas culturales afrolatinoamericanas en diferentes contextos nacionales, así como planes específicos de las agencias transnacionales, como la ONU y la UNESCO. ${ }^{3}$ En este contexto, las acciones emprendidas en el marco de la RdE en Argentina, se conjugan con las reformulaciones locales de las políticas públicas respecto de la cuestión afroargentina que tuvieron un particular impulso a partir de la década de los 2000.

Luego del 2001, en Argentina se abrió una nueva etapa

\footnotetext{
${ }^{3}$ En el marco de la renovada atención de organismos transnacionales hacia la cuestión afro, destaco las declaraciones de la ONU del año 2011 como Año internacional de los afrodescendientes y el Decenio de los Afrodescendientes 2015-2025, al cual Argentina adhiere en 2017 por decreto presidencial (Decreto 658/2017), designando a la Secretaría de Derechos Humanos y Pluralismo Cultural del Ministerio de Justicia y Derechos Humanos como la unidad de coordinación de las acciones a desarrollarse en Argentina.
} 
para la reflexión alrededor de la presencia africana, histórica y actual desde el campo académico y el activismo afrodescendiente, que también involucró diferentes instancias estatales. Algunos autores han analizado las reformulaciones que han atravesado el estado argentino entre los años 2001 y 2015 en términos de políticas de reparación y de visibilización para con la población afrodescendiente, cuando por primera vez se ejecutaron programas estatales ad hoc (López 2006; Fernández Bravo 2012). Ello ocurrió en un contexto internacional caracterizado por las políticas de diversidad y de derechos culturales y por las luchas antirracistas de los movimientos afro de la región latinoamericana (Lao-Montes, 2007; Ferreira 2009; López, 2012). En este contexto de "resurgimiento afro", la nueva agenda de políticas públicas va en dirección del reconocimiento y de la reparación histórica para con la población afrodescendiente en respuesta a las demandas de los grupos organizados de afroargentinos y africanos que se fueron consolidando en esos años (López, 2006; Frigerio y Lamborghini, 2011; Annecchiarico, 2016). ${ }^{4}$ La emergencia de políticas públicas argentinas para con los afrodescendientes son el resultado de una compleja articulación local/global entre los movimientos afro, las políticas nacionales de inclusión social y de diversidad cultural, y las interferencias de las agendas internacionales, especialmente la Conferencia Mundial contra el racismo de Durban del año 2001 con sus recomendaciones (Ferreira, 2009; López, 2012; Annecchiarico 2016). Los estudios afroargentinos consideran las ambivalencias y los desafíos de las políticas de "reconocimiento" y "visibilización" de la presencia africana, teniendo en cuenta los procesos de alterización y de subalternización de memorias, legados culturales y experiencias afrodiaspóricas diferenciales (Frigerio, 2008; López, 2009; Geler, 2010, 2016; Frigerio y Lamborghini, 2011).

Por otro lado, novedosas lecturas sobre la africanidad de las expresiones culturales argentinas, específicamente del área rioplatense, como la murga, la milonga, el tango y el candombe, ponen al centro de la problemática las tensiones racializadas y a la agencia de los sujetos afrodescendientes (Ferreira, 2008; Domínguez, 2009; Parody, 2013; Annecchiarico, Martín y Mercado 2017). Se empezó a reflexionar sobre el patrimonio afroargentino, un tema hasta ese momento prácticamente ausente de las políticas oficiales y de las reflexiones intelectuales. Las actividades realizadas en el marco de la RdE fueron una ocasión para activar el debate respecto del patrimonio cultural afroargentino, conjugando de manera inédita la actividad académica con las políticas del patrimonio sobre la temática, con el objetivo de dar cuenta de la presencia histórica africana y afroargentina a partir de sitios de memoria de la esclavitud y prácticas culturales, como

\footnotetext{
${ }^{4}$ Entre las principales medidas adoptadas en ese período, cito: la inclusión de la variable afrodescendiente en el censo de población del 2010; la creación de programas estatales o áreas especiales para afrodescendientes en el Ministerio de Justicia y en el Ministerio de Cultura; en el 2013, la declaración del 8 de noviembre como el Día Nacional de los Afroargentinos/as y de la Cultura Afro.
}

veremos (UNESCO 2012). A continuación, propongo un recorrido del programa RdE a nivel general, para ver como se organiza y cuales funciones cumple.

\section{La "Ruta del Esclavo" UNESCO en América Latina y el Caribe}

El programa RdE fue lanzado en la $27^{\text {a }}$ Conferencia general de UNESCO en 1993 (Resolución 27 C/3.13) e iniciado en 1994 en Ouidah, Benín, con el impulso de Haití y de algunos países africanos. Es un proyecto administrado, coordinado y supervisado por la División de Políticas Culturales y Diálogo Intercultural de la UNESCO y se inscribe en el marco de las Rutas de Diálogo. ${ }^{5}$ El Comité Científico Internacional, nombrado por el director general de UNESCO tiene como función la de orientar y promover la cooperación internacional entre los grupos de trabajo de cada país y las investigaciones que se desarrollan localmente. ${ }^{6}$ Para América Latina y el Caribe, la RdE fue implementada desde su comienzo en la sede regional de UNESCO de La Habana. En América continental, se registran experiencias más tardías desarrolladas en el marco del programa en Colombia, Perú y Brasil, además que en la región rioplatense objeto de este estudio, como se denomina el espacio geocultural compartido por Argentina, Uruguay y Paraguay?.

El principal propósito de la RdE es el "deber de verdad" sobre la esclavitud y la trata, siendo que poco o nada se ha hecho al respecto a nivel internacional (UNESCO, 2009, 2014). Cabe destacar que, de acuerdo a los documentos del programa RdE revisados, emerge una idea de cultura en tanto "plataforma de dialogo", y como espacio de encuentros, una instancia de "reconciliación gracias en particular al patrimonio cultural"8. La RdE enfrentaría de esta manera una deuda que tenían sobre la temática las organizaciones internacionales, particularmente la ONU y los países miembros, configurándose como un marco legal global para la reparación histórica para con

\footnotetext{
${ }^{5}$ Las Rutas de diálogo tienen el objetivo de describir e investigar algunas trayectorias históricas y culturales relacionadas con la comercialización de productos materiales a lo largo de diferentes regiones del mundo, como la Ruta del Hierro y la Ruta de la Seda, o rutas de intercambios culturales, como la Ruta de El-Andalus en el Mediterráneo y la Ruta del Esclavo.

${ }^{6}$ El Comité Científico Internacional está compuesto por investigadores provenientes de Europa, África y América. Se reúne periódicamente para intercambiar experiencias y avanzar en las diferentes etapas del proyecto. Las reuniones han sido celebradas en: Uidah, Benin 1994; Matanzas, Cuba 1995; Cabinda, Angola 1996; Lisboa, Portugal 1998; Palermo, Italia 2000; Río de Janeiro, Brasil 2001; París, Francia 2006 y 2009; Cartagena, Colombia 2011; Calabar, Nigeria 2012; México 2014; Cabo Verde 2015. Cabe destacar que la historiadora Marta Goldberg es la única argentina que integró dicho Comité. Véase Pineau y Efron (2009).

${ }^{7}$ Acciones y objetivos del proyecto RdE en: www.UNESCO.org/new/ es/culture/themes/ dialogue/the-slave-route. Se pueden consultar también los documentos La Ruta del Esclavo. Reconciliar el deber de memoria con la verdad histórica (UNESCO, 2009) y La Ruta del Esclavo: 1994-2014. El camino recorrido (UNESCO, 2014).

8 Definición que encontramos en: http://www.UNESCO.org/new/es/ culture/themes/dialogue/intercultural-dialogue/ (consultado el 15 de julio de 2017).
} 
la población afrodescendiente y africana.

De acuerdo a los documentos disponibles en la red, podemos ubicar tres etapas principales del proyecto en los primeros veinte años, 1994-2014 (UNESCO, 2014). En la primera etapa, de 1994 a 2005, el proyecto se centró en la investigación científica sobre los distintos aspectos de la esclavitud y la trata transatlántica en el espacio norte atlántico, en un segundo lugar en el Océano Indico y el Mediterráneo. Además, el proyecto promovió la recopilación de documentación histórica relativa a la esclavitud y la trata en diferentes países con el apoyo del proyecto UNESCO "Memoria del Mundo" (UNESCO, 1993). Entre los principales impactos del programa a nivel global para ese período se ubica el aumento de las investigaciones y la inclusión de estos temas en la agenda internacional ${ }^{9}$.

En el año 2006, la Nueva estrategia para la Ruta del Esclavo propone algunas reformulaciones, cuales: inclusión de nuevos temas y ampliación geográfica; estudio de las consecuencias de la esclavitud, las formas de resistencia y las nuevas formas de esclavitud (UNESCO, 2006). El principal aspecto a destacar en esta segunda etapa del programa, es la inclusión de la lucha contra el racismo como eje de trabajo, aspecto ausente en las primeras orientaciones. ${ }^{10}$ Encontramos además un interés hacia el patrimonio cultural inmaterial en cuanto "portador de huellas que no se deben borrar", entendiendo que "el patrimonio resultante de la trata esclavista y de la esclavitud es a la vez material e inmaterial" y que "las expresiones artísticas siguen presentes en el centro de las culturas vivas de los pueblos" (ibid). ${ }^{11}$ Es en esa etapa del programa que Argentina, junto a Uruguay y Paraguay, inician sus primeras acciones en el marco de la RdE (Pineau y Efron 2009). Entre 2008 y 2013 se registraron para América Latina y el Caribe 16 expresiones culturales vinculadas con el legado africano declaradas Patrimonio Cultural Inmaterial de la Humanidad, en tanto "elementos característicos del mestizaje cultural consiguiente a la trata negrera" (UNESCO, 2014, p. 10). Entre ellas, se encuentran el Tango y el Candombe (ibid).

Entre 2011 y 2012 la RdE atraviesa la crisis internacional financiera que implicó una drástica reducción de los presupuestos de UNESCO, lo cual tuvo efectos también en la trayectoria del programa en Argentina, como veremos. ${ }^{12}$

\footnotetext{
${ }_{9}^{9}$ Declaración de la trata transatlántica y la esclavitud como crimen de lesa humanidad en la Conferencia Mundial de Durban en 2001; proclamación del 23 de agosto Día Internacional del Recuerdo de la Trata de Esclavos y su Abolición y el año 2004 Año Internacional del Conmemoración de la Lucha contra la Esclavitud y su Abolición.

10 La Asamblea General de Naciones Unidas en su $61^{\text {a }}$ reunión de 2006, conmemoró el bicentenario de la abolición de la trata transatlántica y declaró el 27 de marzo de 2007 el día internacional de dicha conmemoración.

11 Estas reformulaciones reflejan lo establecido en la Convención para la Salvaguardia del Patrimonio Cultural Inmaterial de 2003 y la Convención sobre la Protección y Promoción de la Diversidad de las Expresiones Culturales de 2005.

12 Se puede consultar por ejemplo la carta enviada a la directora de UNESCO Irina Bokova por la Presidenta del Comité Científico
}

En este contexto, se realizan nuevas reformulaciones del programa, que pasa a llamarse La Ruta del Esclavo. Resistencia, Libertad, Patrimonio. Los conceptos añadidos en el subtítulo - resistencia, libertad y patrimonio - , marcan en términos generales un reposicionamiento de la RdE respecto de sus objetivos y estrategias macro. Por un lado, se orienta a la visibilización de las formas de resistencia de las comunidades afrodescendientes a lo largo de la historia, con una atención específica al tema del patrimonio y, por otro lado, a la vigencia del racismo y la necesidad de enfrentarlo con medidas de políticas públicas nacionales. En efecto, en la reunión del Comité Científico Internacional realizada en el año 2012 en Nigeria, se recomienda, entre otras cosas: la creación de un observatorio estadístico sobre afrodescendientes en América Latina el Caribe; sugerir a los países la institución de días internacionales y nacionales de los afrodescendientes; acciones y estrategias para la lucha contra la discriminación y el racismo; la enseñanza y la elaboración de nuevos materiales pedagógicos (Scientific Committee of de UNESCO Slave Route Project, 2012). Estas recomendaciones van de la mano de la Conferencia Mundial contra el Racismo celebrada en Durban en el año 2001, donde se invitaban a los Estados signatarios a "formular políticas públicas destinadas a esa población en relación con la deuda resultante del proceso esclavista". ${ }^{13}$ Estas recomendaciones se conjugan además con las políticas públicas en temática afro implementadas en Argentina luego del 2001, lo cual marca una interconexión entre las agendas globales y locales en la elaboración de medidas de reparación para con la población afrodescendiente.

\section{Argentina y el espacio atlántico de la esclavitud}

En su comienzo el proyecto la RdE fue pensado para la investigación histórica sobre la trata transatlántica y la esclavitud en regiones que podríamos circunscribir al Black Atlantic propuesto por Gilroy (2003 [1993]): Estados Unidos, Caribe, Europa y África occidental. Es para mediados de los 2000, que Argentina, junto a Paraguay y Uruguay, se sumó al programa, con el objetivo de recuperar la memoria de un pasado común que ha sido ignorado o negado durante mucho tiempo, de acuerdo a lo expresado en los documentos del primer encuentro regional realizado en Montevideo en el año 2004 (UNESCO 2005). Es posible argumentar que esta llegada tardía es un reflejo del estado de los debates sobre la esclavitud y la trata a nivel regional que empezaban

Internacional María Elisa Velázquez Gutiérrez, donde expresa su preocupación frente a la situación financiera que afecta las actividades del Comité Científico Internacional. (Velázquez Gutiérrez 2012). Además, en el informe del Comité Científico Internacional reunido en Nigeria en 2012, se destaca la necesidad de obtener mayores recursos para garantizar la continuidad de las acciones emprendidas por el programa (Scientific Committee of de UNESCO Slave Route Project, 2012).

13 Informe de la Conferencia Mundial contra el Racismo, la Discriminación Racial, la Xenofobia y las Formas Conexas de Intolerancia, Durban, 31 de agosto al 8 de septiembre de 2001, A/ CONF.189/12, pag. 11 
a consolidarse y a circular para esos años. Además, ello da cuenta de una cierta marginalidad en la cartografía afroamericana según los organismos internacionales, que tiene sus centros lejos de los puertos de Buenos Aires y Montevideo ${ }^{14}$.

Es posible relevar un primer proyecto realizado en el marco de la RdE entre los años 2002 y 2003, cuando el Archivo General de la Nación Argentina, gracias a una financiación del proyecto Memoria del Mundo de UNESCO, digitalizó documentación relativa a la esclavitud y el tráfico de africanos esclavizados en época colonial. El archivo digitalizado cuenta con un total de 500 documentos que abarcan el período de 1563 a 1821 y se refieren al contexto geográfico del Virreinato del Río de la Plata: Argentina, Uruguay, Paraguay, Brasil, Bolivia y Perú (Alonso, 2002-2003). El objetivo de dicha digitalización es la conservación de la mayor cantidad de documentos existentes sobre la esclavitud en Argentina, para su accesibilidad pública (Alonso, 2005: 47). Este proyecto, novedoso e inédito para Argentina, se configura como una respuesta local a los objetivos globales de la $\mathrm{RdE}$ que, como vimos, se centraron en un primer momento en la promoción - y financiación - de la recopilación de fuentes documentales y la investigación histórica sobre la trata transatlántica de africanos esclavizados (UNESCO 2014). Este archivo es una herramienta fundamental para la investigación historiográfica, más aún sobre un tema que está ausente del imaginario común. ${ }^{15}$ En efecto, tuvo un cierto eco mediático en la opinión pública que es posible relevar a través de la prensa. Por ejemplo, una nota publicada en 2003 en el diario la Nación, uno de los principales periódicos argentinos, expresa que

sumergirse en el mundo de los esclavos del Río de la Plata es descubrir, por ejemplo, que la pintoresca mazamorrera que los chicos representan en los actos escolares del 25 de Mayo era -horror- una esclava que trabajaba probablemente para una señora viuda. Y que en cuanto bajó del barco fue marcada a hierro caliente -como el ganado- y separada de los suyos ${ }^{16}$.

Esta cita nos permite destacar algunas ambivalencias en la conformación del archivo respecto de la narrativa hegemónica nacional sobre la esclavitud y los afroargentinos. En primer lugar, el recorte temporal elegido en el marco del proyecto de digitalización mencionado, no considera la época poscolonial, cuando la institución de la esclavitud siguió operando ${ }^{17}$. Podemos observar que esta

\footnotetext{
14 Roberto Pacheco considera que la existencia de una hegemonía anglosajona define "los parámetros del estudio de lo afro, reduciendo Afroamérica al Caribe y a Brasil" es decir una geolocalización escencializada y acotada de la presencia afro en América, en la cual no cabría la región rioplatense (Pacheco 2008: 12-13).

${ }^{15}$ Aclaro que no pude indagar etnográficamente el uso de este archivo por parte de investigadores, siendo esta una tarea que merece ser realizada.

${ }^{16}$ Nota "Reconstruyen la ruta de los esclavos en la época colonial" por Fernando Halperin, Diario La Nación, del 17 de mayo de 2003.

${ }^{17}$ En Argentina, la abolición de la esclavitud fue un proceso gradual que duró más de 40 años. La Asamblea del Año XIII decretó la libertad
}

periodización ubica la presencia africana y afroargentina exclusivamente en el pasado colonial, avalando así la idea de la desaparición de la población afroargentina en la época republicana (Andrews 1989). Como han puesto en evidencia investigadores contemporáneos, la idea de desaparición de los afroargentinos se basa en una construcción discursiva hegemónica de silenciamiento e invisibilización de la presencia afroargentina que es pensable, si a caso, únicamente al pasado colonial y la esclavitud y de manera estereotipada (Picotti, 2001; Geler, 2010). ${ }^{18}$ Este discurso ideológicamente construido fue avalando de alguna manera el "enigma de la desaparición" de los afroargentinos, particularmente en los censos y en los estudios historiográficos (Andrews 1989). En efecto, los principales trabajos historiográficos sobre la temática refieren a la época colonial, siendo escasos los trabajos dedicados a los afroargentinos en la segunda mitad del siglo XIX y más aún los que tratan aspectos de la presencia afroargentina durante el siglo XX (Frigerio, 2008; Pacheco, 2008). Al respecto, la investigadora argentina Liboeiro (1999) habla de una mirada racista persistente que condiciona la producción sobre la presencia afro en Argentina:

no es fácil hacer evidente para la Historia y los argentinos, una realidad tan afanosamente escondida por un voluntarismo racista y elitista (...) el prejuicio hacia la negritud ha desviado los estudios creyendo que no serían aceptados. Los "motivos" técnicos - como la falta de documentos, comunidades actuales pequeñas en relación a la población general- no son suficientes como para no aclarar la nebulosa tendida sobre este tema en nuestro país (Liboeiro, 1999: 42)

La esclavitud y las luchas por la abolición, fueron olvidadas en las historiografías nacionales porque son historias impensables, hechos discordantes, verdades incómodas que las naciones - y las disciplinas - no pudieron enfrentar debidamente (Trouillot 1995). Frente a ello, el Archivo permite replantear la centralidad de ese proceso en la conformación de la nación Argentina, en contra de su silenciamiento y olvido en los discursos pedagógicos de nación (Bhabha 2002).

\footnotetext{
a los hijos de las mujeres esclavizadas que nacieran después del 31 de enero de 1813. El decreto además preveía que todo esclavizado que pisara suelo argentino, era libre. Sin embargo, esa medida no acabó con la esclavitud de africanos y afroargentinos. La abolición de la esclavitud recién se declaró en el artículo 15 de la Constitución de la Confederación Argentina de 1853, y en la provincia de Buenos Aires se instrumentó luego de 1861. El tráfico esclavista había sido prohibido en el 1813, pero hasta mediados del siglo XIX continuó la comercialización de africanos y africanas en territorio argentino, incluso después del tratado antiesclavista de Gran Bretaña de 1840 (Cfr. Andrews, 1989).

${ }^{18}$ Los actos escolares del 25 de mayo - que conmemoran la Revolución de Mayo de 1810 - dan cuenta de una fijación estereotipante de los afroargentinos en papeles marginales y folklorizantes del pasado. Mientras que ya para la conmemoración del día de la independencia del 9 de julio 1816, los afrodescendientes desaparecen del imaginario nacional y de las representaciones escolares.
} 


\section{Trayectorias locales de la Ruta del Esclavo UNESCO}

El primer encuentro regional de la RdE en el Río de la Plata se realizó en el año 2004, en Montevideo, con el simposio internacional La Ruta del Esclavo en el Río de la Plata: su historia y sus consecuencias (UNESCO, 2005). El evento, organizado por la Oficina UNESCO para Mercosur, se desarrolló en ocasión del Año Internacional de Conmemoración de la Lucha Contra La Esclavitud y de su Abolición. Se reunieron diferentes investigadores, funcionarios y miembros de la sociedad civil de Argentina, Uruguay, Brasil y Paraguay, así como referentes del movimiento afrouruguayo, para discutir sobre diferentes aspectos de la esclavitud en la región, aportando "contribuciones sobre la investigación histórica, la situación actual social y cultural de la población afrodescendiente y proyecciones para un mundo sin racismo y exclusión social" (UNESCO, 2005: 7). Estas consideraciones reflejan por un lado los objetivos y enfoques que se formalizaron en la Nueva Estrategia del año 2006. Por el otro, responden a las expectativas y demandas de inclusión y lucha contra el racismo del activismo afrodescendiente de la región. Un dato a destacar en este sentido es la participación, además que de investigadores y funcionarios de la UNESCO, de representantes de organizaciones afrouruguayas: Mundo Afro y su Director General Romero Jorge Rodríguez; el Centro Cultural por la Paz y la Integración dirigido por Beatriz Santos Arrascaeta; la Unidad temática de afrodescendientes de la intendencia de Montevideo. Entre los expositores argentinos figuran Gustavo Fabián Alonso, Coordinador General del Área de Digitalización del Archivo General de la Nación de Argentina, y dos reconocidas investigadoras, Marta Goldberg y Silvia Mallo.

A raíz de este encuentro, en Argentina se activó una red de personas que tomaron el proyecto a partir de una propuesta del Sector Cultura de la Oficina UNESCO de Montevideo a cargo de Fréderich Vacheron, que recién acababa su servicio en la Oficina UNESCO de La Habana y desde allí venía cargado de mucho entusiasmo (Fréderic Vacheron, entrevista, Buenos Aires, agosto de 2014). Con el impulso de la diplomática Susana Pataro, se constituyó una red con la oficina de Relaciones exteriores de Argentina, la Cátedra UNESCO de Turismo Cultural de la Universidad de Tres de Febrero de Buenos Aires y la Asociación de Amigos del Museo Nacional de Bellas Artes. Estos actores decidieron tomar el proyecto y organizar un primer encuentro de alcance internacional con sede en Buenos Aires (Marisa Pineau, entrevista, Buenos Aires, enero de 2014).

En octubre de 2009 y de 2010 se realizaron dos seminarios internacionales y dos talleres de sitios de memoria, coordinados por Marisa Pineau, historiadora y coordinadora de la Sección de Estudios Asia y África de la Universidad de Buenos Aires. Tuvieron financiación de la Agencia Española de Cooperación Internacional para el Desarrollo (AECID) y con el auspicio del Ministerio de
Relaciones Exteriores, Comercio Internacional y Culto de la República Argentina, de la Representación de la UNESCO ante el Mercosur de Montevideo y de la Embajada de los Estados Unidos en la Argentina. En los dos seminarios, gratuitos y abiertos al público, participaron investigadores, funcionarios públicos y de organismos internacionales y referentes de organizaciones afro de la región.

El primer simposio internacional La Ruta del Esclavo en el Río de la Plata. Aportes para el diálogo intercultural del 2009 se desarrolló durante dos días, en la sede de la Asociación de Amigos de Museo Nacional de Bellas Artes, con alrededor de 30 oradores, principalmente académicos e investigadores, diplomáticos y representantes de organismos internacionales involucrados en el proyecto (Pineau y Efron, 2009). Uno de los objetivos de los eventos fue conocer la experiencia desarrollada en el Caribe Latino, particularmente en Cuba y Haití, que pudiera servir como ejemplo virtuoso de gestión del proyecto. Hubo investigadores de distintos países de América Latina, de África y de Europa, convocados con el objetivo de "hacer una política fuerte de acercamiento entre los continentes que tiene que estar acompañado por el tema cultural, en esa idea de diálogo intercultural" (Marisa Pineau, entrevista, Buenos Aires, enero de 2014). Marisa Pineau comenta que este primer seminario fue una experiencia exitosa que demostró que por un lado había interés en esos temas y por el otro que había necesidad de visibilizar no solamente la presencia africana tan negada en la región, sino también las investigaciones y los trabajos que ya se estaban realizando y que tenían poca difusión (Marisa Pineau, entrevista, Buenos Aires, enero de 2014).

Concluida esta experiencia, la misma red de personas e instituciones determinó realizar en octubre de 2010, el segundo simposio internacional, Huellas y legados de la esclavitud en las Américas. Vale la pena destacar la coyuntura nacional de ese año 2010, que coincidió con las celebraciones del bicentenario de la República Argentina y con el censo de población que incluyó por primera vez la variable afrodescendencia. A su vez, el seminario quiso rendir un doble homenaje, tal como expresado en el folleto explicativo: al 60 aniversario de las independencias de las naciones africanas y a Haití, por su trascendencia histórica en las luchas antiesclavistas e independentistas de América y además por las consecuencias trágicas del terremoto de ese mismo año. Además, las actividades se enmarcaron en el año 2010, año Internacional del Acercamiento de las Culturas, anticipando el año 2011, declarado año Internacional de los afrodescendientes por la ONU. Podemos argumentar entonces que estos cruces entre las plataformas globales y los escenarios locales, sirvieron para otorgar una cierta legitimidad a la RdE en línea con una visión multiculturalista de lo políticamente correcto y a su vez fundamentar su ejecución en Argentina, que se plantaba, junto a los otros países de la región, como un nuevo centro de reflexión y de producción de conocimiento sobre la temática. Hay que destacar que ambos encuentros, referentes del 
movimiento afroargentino hablaron de la invisibilización histórica de la población afroargentina, destacando el papel de las organizaciones en la lucha contra el racismo y las demandas de inclusión en las agendas públicas del Estado (Gomes, 2011; Álvarez Nazareno, 2012; Pita, 2012).

A pesar de estos encuentros y actividades, la trayectoria local de la RdE no tuvo continuidad, las agencias financiadoras no renovaron sus aportes, en un contexto de crisis financiera global. Prácticamente ninguna actividad se desarrolló posteriormente al 2010, excepto la publicación de los resultados de los seminarios y talleres de 2009 y 2010 (Pineau 2011, 2012; UNESCO 2012), y la celebración de los veinte años de la RdE en 2014. ${ }_{19} \mathrm{Si}$ bien entre las recomendaciones de la RdE figura la creación de comités nacionales que den seguimiento a las actividades a nivel local, en Argentina no se conformó un grupo de trabajo nacional ${ }^{20}$.

\section{Sitios de memoria de la esclavitud: construyendo legitimidades}

Paralelamente a los dos seminarios, se realizaron dos ediciones del Taller de Sitios de Memoria de La Ruta del Esclavo en Argentina, Paraguay y Uruguay en Buenos Aires. Esta actividad responde a la directiva de la nueva estrategia de la RdE de realizar inventarios para la preservación del patrimonio material y sitios de memoria (UNESCO 2006). Especialistas de los tres países presentaron doce sitios documentados según los criterios de la UNESCO, cuatro para Argentina, tres para Paraguay y cinco para Uruguay (UNESCO, 2012). Para Argentina se incluyeron: Plaza San Martín y Parque Lezama en la ciudad de Buenos Aires; la Capilla de los Negros en Chascomús en la provincia de Buenos Aires; y la Manzana y las Estancias jesuíticas de Córdoba; un conjunto de expresiones culturales con el título de "Candombe, milonga, tango y payadas en el espacio cultural afrorioplatense de la ciudad de Buenos Aires" (UNESCO, 2012)21.

Esta tarea, sin precedentes al no existir una estrategia regional ni nacional para trabajar en ese sentido, fue producto de una articulación entre investigadores, agencias privadas y representantes de organizaciones afrodescendientes.

\footnotetext{
${ }^{19}$ Se puede ver la programación de la actividad por los veinte años del programa en: www.UNESCO.org/new/es/media-services/singleview-tv- release/news/argentina_activities_for_the_20_years_of_the_ project_slave_route/ (consultado el 15 de julio de 2017).

20 Hay que decir que en Córdoba se consolidó en 2010 un grupo autodenominado "Ruta del Esclavo. Grupo Córdoba" que realiza hasta la fecha actividades de divulgación sobre temáticas afroargentinas a nivel local. El grupo está conformado por investigadores, activistas afrodescendientes y representantes de instituciones locales de cultura y patrimonio, particularmente vinculados al sistema de las estancias jesuíticas. https://rutadelesclavocba.wordpress.com/ (consultado el 15 de mayo de 2018).

${ }^{21}$ Los informes fueron realizados respectivamente por Marta Goldberg, Dina Picotti, Rebeca Medina y Monica Lacarrieu, reconocidas investigadoras relacionadas con los estudios afroargentinos o los estudios de patrimonio. (UNESCO, 2012).
}

Podemos considerar que los tres sitios y el conjunto de expresiones culturales escogidos dan cuenta de una variedad histórica, geográfica y antropológica de la presencia africana en la Argentina, si bien no sean los únicos. Marisa Pineau expresa que se eligieron esos sitios porque son los más conocidos o, por lo menos, los más documentados. Pero sobre todo, había poco tiempo para realizar la investigación, con lo cual se priorizó realizar una primera recopilación "que sirviera en un futuro como material trabajado bajo los cánones de la UNESCO para que los países los asumieran como sitios de memoria propia y quizás, como objetivo a largo plazo, alguno de ellos llegara a ser Patrimonio de la Humanidad" (Marisa Pineau, entrevista, Buenos Aires, enero de 2014). Estos sitios escogidos responden a los requisitos de UNESCO, en tanto sitios de memoria materiales, inmateriales o mixtos con ubicación geográfica, antecedentes históricos, sociales, culturales, su grado de preservación y su apropiación por parte de la comunidad en la que están insertos (UNESCO, 2012). Otro requisito UNESCO que Marisa Pineau destaca es la participación de las comunidades en los proyectos: el aval de las comunidades es fundamental para dar legitimidad a cualquier acción emprendida por las instituciones (Marisa Pineau, entrevista, Buenos Aires, enero de 2014). En los Talleres de Sitios de Memoria, se convocaron a representantes de organizaciones de afrodescendientes, Miriam Gomes de la Asociación Caboverdiana de Dock Sur y Federico Pita de DIAFAR, para articular con los demás actores con el fin de construir una plataforma de consenso (ibid). Sin embargo, en el caso analizado de la Capilla de los Negros de Chascomús que veremos a continuación, la participación de representantes de organizaciones afro en las mesas de diálogo propiciadas por los Talleres, no se acompañó de una real inclusión de la comunidad afro de Chascomús a lo largo del proceso patrimonial.

De estas consideraciones preliminares, se desprende una delicada disputa por la legitimidad de acuerdo a posicionamientos diferenciales de los actores involucrados en los procesos de patrimonialización. Una cuestión importante refiere a como representar y como dar cuenta de lo que fue la esclavitud y sus consecuencias, particularmente el racismo, a partir de las miradas, usos y sentidos otorgados a los bienes patrimoniales por parte de diferentes agentes. En otras palabras, se trata de poner en debate si la legitimidad de sitios de memoria de la esclavitud viene del ámbito académico e institucional o viene del saber de las comunidades, o si de ambos. Un segundo aspecto para destacar es relevar cómo se traduce la participación y representatividad de las comunidades en todas las fases de los proyectos de patrimonialización, especialmente las investigaciones, la sociabilización de los resultados, la gestión y la restitución patrimonial (Cruces 1998).

De acuerdo a estas inquietudes, propongo abordar la experiencia de la Capilla de Los Negros de Chascomús, caso que he analizado en trabajos recientes (Annecchiarico, 
2014, 2016). El sitio se destaca por diferentes factores que hacen a su singularidad: en primer lugar, se trata del único monumento nacional y provincial de la provincia de Buenos Aires, que refiere a la presencia histórica y actual de la población de origen africano desde la época colonial (Schávelzon, 2003; Carré y Lagreca, 1998). En segundo lugar, la Capilla de los Negros es un sitio de religiosidad popular mantenido por la familia descendiente de sus antiguos fundadores de mediados del siglo XIX, la Cofradía de la Hermandad de Morenos Bayonbé de Invensa, y por eso es considerada por el activismo afroargentino un referente de la presencia y de la agencia afroargentina no solamente en el pasado, sino sobre todo en el presente.

\section{La Capilla de los Negros de Chascomús}

La Capilla de los Negros de Chascomús, ubicada en la provincia de Buenos Aires, fue constuida en 1862 por la Hermandad de Morenos Bayonbe de Invensa. Fue declarada en 1962 Lugar Histórico Nacional y en 1992 Sitio Histórico Provincial. En 2011 fue nombrada sitio histórico de la memoria de la Ruta del Esclavo en el Río de la Plata, tal como se lee en el certificado expuesto en el interior de la Capilla y en la placa colocada en la entrada. Es el único sitio de la provincia de Buenos Aires que tiene un reconocimiento internacional de la magnitud de UNESCO 22 .

La Capilla de los Negros es un espacio de religiosidad popular sincrética abierto a todos los que quieran visitarla y dejar su ofrenda, mantenida y cuidada generación tras generación por los descendientes de uno de los fundadores, Luciano Soler, persona destacada en la memoria familiar y local. ${ }^{23}$ Hoy sigue siendo un lugar de encuentro y de activismo afro a través de la cultura, en donde se realizan celebraciones religiosas, artísticas y actividades políticas, lo cual la convierte en un símbolo de agencia de la comunidad afroargentina. Su estructura es en gran parte originaria de la época. Las paredes son de mampostería y el piso de tierra pisada; el techo de chapa y una cruz han sido colocados en los años 50 luego que un fuerte temporal tumbara el techo original (Carré y Lagrega, 1998). En su interior, se encuentra un altar; allí y en las paredes, cohabitan santos de la religión católica, santos populares argentinos no canonizados, próceres y personajes históricos, deidades de las religiones afroamericanas, una partitura con letra de una milonga titulada "Capilla de los morenos". Se destacan además

\footnotetext{
22 La placa de madera colocada en la entrada de la Capilla dice: "Capilla de los Negros. Designado por la UNESCO integrante de la Ruta del Esclavo del Río de la Plata. Monumento histórico nacional, construido en el año 1862 con características religiosas propias que mezclaban rituales africanos y candombes rioplatenses. Fue utilizada como lazareto en las epidemias de los años 1868 y $1871^{\prime \prime}$.

${ }^{23}$ Conocido también como Luciano Alsina, el apellido de la familia de hacendados para quienes trabajaba. Fue además fundador de una comparsa de carnaval muy conocida, Los Negros Alegres, que estuvo activa hasta mediados de los años Veinte, de acuerdo a testimonios recopilados en periódicos de la época, notas de escritores e historiadores locales.
}

recortes de notas periodísticas de diferentes épocas sobre la Capilla e imágenes - fotografías, retratos, dibujos - de su cuidadora más célebre, Doña Eloísa Soler, bisnieta de Luciano Soler, fallecida en los años noventa del siglo XX24.

En una entrevista realizada para un periódico local a finales de los años '70, Doña Eloísa, dice: "Este es el templo de los morenos. La construyeron mis bisabuelos y siempre alguien de la familia estará para cuidarla". ${ }^{25}$ Antonio Luis, hijo de Doña Eloísa, siguió la tarea del cuidado de la Capilla, abriéndola al público, manteniéndola limpia y con velas siempre encendidas. "El día que Antonio ya no esté es obligación nuestra seguir cuidándola", afirma Soledad Luis, sobrina de Antonio y nieta de Doña Eloísa, que declara seguir con orgullo el mandato familiar (Soledad Luis, programa de la Televisión Pública "Vivo en Argentina", 24/11/2012, disponible online). Soledad es guía de turismo de la municipalidad y trabaja para visibilizar la historia de los afrodescendientes de Chascomús: "la Capilla es nuestro punto de referencia, aquí podemos mostrar la verdad, o sea, que aquí estamos" (Soledad Luis en Luongo, 2013). A su vez, denuncia una historia de invisibilización y de discriminación sufrida por la comunidad afrochascomunense, compuesta por su familia y otras familias descendientes de los africanos que trabajaron como esclavizados en la localidad. Particularmente, argumenta que existe un interés ambivalente por parte de la municipalidad en valorizar la Capilla y una visión parcial y estereotipada de la historia afro en la bibliografía local (Soledad Luis, conversaciones, Buenos Aires, abril de 2015).

Si bien la Capilla de los Negros esté incluida en el recorrido turístico ofrecido por la municipalidad, sea patrimonio Nacional y Provincial además que sitio de memoria de la esclavitud, la presencia afrodescendiente en la historia de la localidad está prácticamente ausente del relato oficial. Ello se observa de manera ejemplar en el Museo Pampeano y en la Casa de Casco, edificios históricos emblemáticos de la ciudad que visité en mi trabajo de campo. En el primer caso, el único elemento alusivo a la población afrodescendiente es un retrato de Ana Escribano, africana esclavizada que trabajó para la familia Escribano, quien participó en la fundación de Chascomús, en 1779. En el segundo caso, la Casa de Casco, de principio del siglo XIX y donde se encuentra el Archivo Municipal y Dirección de Patrimonio Histórico y Cultural, no figura ninguna descripción o alusión concreta al trabajo de esclavizados al servicio de la familia Casco ni tampoco en la historia local.

Por otro lado, se destaca un pequeño corpus de trabajos realizados por historiadores y escritores locales (Luzian, 1953; Isusi, 1953) y sobre todo los textos de Lahourcade

\footnotetext{
${ }^{24}$ La descripción ofrecida en este artículo corresponde a la última visita realizada por mi a finales de 2014, cuando Antonio Luis, hijo de Doña Eloisa, aún se encontraba al frente de la Capilla, hasta su fallecimiento en el año 2015.

${ }^{25}$ Nota "Eloisa la morena de la Capilla", s/a. El Argentino, Chascomús, s/f. Copia conservada en el Archivo Histórico Municipal.
} 
(1973, 2008), quién más se ha dedicado a escribir sobre la historia de la comunidad afro y la Capilla. Especialmente en estos últimos, se traza una parábola necrológica de la Capilla y de la comunidad afro de acuerdo al "lento pero inexorable ocaso de los morenos en Chascomús" (Lahourcade, 2008: 121). La Capilla sería hoy el "único testimonio material relevante (...) envuelta en la tristeza de lo que ya fue, es la reliquia única que recuerda a la raza africana en suelo argentino" (Lahourcade 2008:105, 128). La comunidad afrochascomunenese habría desaparecido con la muerte de Doña Eloísa en 1990, definida como la "última africana pura" (Lahourcade, 2008: 121). Una expresión también retomada en el informe realizado en el marco de la RdE por Picotti (2012:223). ${ }^{26}$ Esta narrativa hegemónica, está atravesada por tensiones racializadas en la construcción de conocimiento. Los afrodescendientes, particularmente los cuidadores de la Capilla, no aparecen como interlocutores, son testimonios mudos, ubicados y pensables únicamente en relación al pasado, respondiendo así al proceso de invisibilización de la negritud a nivel nacional.

En el proceso de patrimonialización de la Capilla en el marco de la RdE se evidencian las ambivalencias de la narrativa hegemónica y la emergencia de una narrativa subalterna. Como vimos, para los Talleres de Sitios de Memoria de la Esclavitud realizados en Buenos Aires en 2009 y 2010, se convocaron a investigadores expertos y a representantes de organizaciones afroargentinas. La investigadora Dina Picotti elaboró el informe final sobre la Capilla de los Negros (Picotti, 2012). Para ello, se realizó un trabajo interinstitucional con la Dirección de Patrimonio Histórico y Cultural de la Municipalidad de Chascomús, que de redactó un informe previo con las principales características del sitio, específicamente a lo que refiere a su importancia para la identidad local, sosteniendo el interés de la Dirección de Patrimonio "para que los afrodescendientes ocupen definitivamente su lugar en la historia de Chascomús" (Bilbao et al. s/f: 4) ${ }^{27}$.

En estos trabajos, pude relevar algunas limitaciones en cuanto a enfoques y metodologías que reproducen una mirada reificadora y estereotipante de la población afrodescendiente, a menudo en línea con la narrativa hegemónica antedicha. Si bien se trate de sistematizaciones interesantes, detalladas y actualizadas sobre la Capilla y su historia, estos informes emplean una metodología de trabajo que no toma en cuenta la agencia de la comunidad afrodescendiente. Esto resulta llamativo si se considera que entre los requisitos de Unesco - la RdE en nuestro caso - se requiere la participación de las comunidades locales en el proceso de patrimonialización. Al respecto,

\footnotetext{
${ }^{26}$ Las contradicciones de este discurso - Eloísa es argentina, no africana; los descendientes siguen al mando de la Capilla; la Capilla es un sitio que sigue en función - y las visiones racializadas de los sujetos y de la otredad sobre la base de un cierto saber "científico" de "pureza racial", dan para un análisis que excede este trabajo.

${ }^{27}$ Pude acceder a este informe en mi estadía de trabajo de campo de 2014 cuando revisé los materiales sobre la Capilla y la comunidad afro conservados en el archivo.
}

Soledad subraya que ella u otro miembro de la familia no fueron involucrados, la Dirección de Patrimonio de la Municipalidad fue la única interlocutora avalada (Bilbao et al. s/f). "Nos enteramos el día que vinieron a la Capilla a colocar la placa [de sitio de memoria la RdE], nadie vino a vernos antes" (Soledad Luis, Seminario "Comunidad afrochascomunense", DIAFAR, Buenos Aires 11 de abril de 2015). En dicho proceso, toman fuerza las disputas por la legitimidad de las voces desigualmente posicionadas.

Como sostiene Marisa Pineau, la RdE es "un marco legal y académico que puede servir para impulsar otro tipo de acciones que lleven adelante las organizaciones"; obtenida esta importante declaratoria, se espera que las organizaciones le den legitimidad, en cuanto "ellos son los portadores" y "reclamar lo que les corresponde" (Marisa Pineau, entrevista, Buenos Aires, febrero de 2014). Cabe destacar entonces las oportunidades movilizadas por la experiencia de patrimonialización de la Capilla en el marco de la RdE para la familia cuidadora. A raíz de esta experiencia, Soledad entró en contacto con el activismo afroargentino. Federico Pita, activista afroargentino y presidente de la DIAFAR, conoció la Capilla por su participación en el Taller de Sitios de Memoria organizado por el proyecto RdE en 2010, tal como contó en un conversatorio público (Federico Pita, Panel "Patrimonio Cultural Afroargentino: estado de la Cuestión", Museo Etnográfico Ambrosetti, Buenos Aires, julio de 2014). La primera y más significativa actividad realizada con la participación de DIAFAR, fue el festival Una vela para la Capilla de los Negros en conmemoración del $150^{\circ}$ aniversario de su fundación, en febrero de 2012, una emotiva ceremonia de encendido de velas, con la actuación de artistas, toques de candombe y charlas. Como destaca Federico (ibid), el objetivo de estas actividades no es solo la puesta en valor del sitio con propuestas culturales y la difusión de la historia de la Capilla por parte de sus protagonistas, sino también difundir el compromiso de la comunidad afroargentina por la lucha contra el racismo. ${ }^{28}$

\section{Consideraciones finales}

El artículo presentó un análisis del proceso de emergencia del proyecto de la RdE UNESCO, su apropiación y puesta en marcha en Argentina, ejemplificando en el caso de la Capilla de los Negros de Chascomús. Para ubicar el análisis, presentamos el contexto local respecto de la cuestión afrodescendiente y la conformación de una agenda política pública específica a partir de los años 2000. Luego, esbozamos un breve recorrido de la trayectoria de la RdE para caracterizar con mas precisión su implementación en Argentina, destacando las

\footnotetext{
${ }^{28}$ Cabe mencionar el video clip "Acá estamos" realizado por DIAFAR, parte de la campaña internacional "Rap contra el racismo" promovida por el artista y activista afro-español El Chojín. Allí aparecen imágenes de la Capilla tomadas durante el Festival, Soledad Luis con Fidel Nadal y un retrato de Doña Eloisa conservada en el altar. El video se puede ver en: www.youtube.com/watch?v=WmXHMt4bgPQ (consultado el 25 de julio de 2017).
} 
actividades realizadas entre 2009 y 2014.

En el caso de la Capilla de los Negros de Chascomús, las articulaciones entre investigadores e instituciones locales por un lado, familias afrodescendientes y activismo político afroargentino por el otro, abrieron un nuevo capítulo en la historia local. El proceso de patrimonialización en el marco de la RdE pone de manifiesto las divergencias y las negociaciones entre narrativas a menudo en conflicto o antagónicas. En el análisis, se pusieron en evidencia dos aspectos centrales: por un lado, el contraste entre el accionar de la familia cuidadora de la Capilla, y el relato de invisibilización o una narrativa del silencio (Trouillot 1995) producida por la bibliografía disponible; por el otro, la Capilla como un archivo vivo en donde lo material y lo inmaterial se conjugan de manera articulada y dinámica, y para cuya significación intervienen diferentes actores que disputan su representación en el marco local y nacional.

Para finalizar, me interesa plantear algunas interrogantes para pensar las ambivalencias y los desafíos de la RdE desde una perspectiva local de acuerdo a la experiencia de la Capilla de los Negros. A partir de nuestro análisis, emergen narrativas, memorias y usos del pasado en tensión. En tanto sitio de memoria de la esclavitud, la Capilla de los Negros es objeto de negociaciones entre diferentes sujetos y narrativas a menudo en conflicto o antagónicas. Por un lado, las declaraciones nacionales, provinciales e internacionales construyen significados alrededor de la Capilla: monumento histórico, sitio de memoria de la esclavitud. Es decir, el proceso de patrimonialización de la Capilla iniciado a mediados del siglo $X X$, reifica su espacio, lo monumentaliza, lo cristaliza en un pasado negro pensable únicamente en relación a la esclavitud, de acuerdo a la producción historiográfica dominante, y no considera las formas de transmisión de la memoria que los sujetos - principalmente la familia cuidadora - realizan. Por otro lado, el constante y minucioso trabajo de los que habitaron y habitan los espacios físicos y simbólicos de la Capilla, elaboran otras narrativas sobre la afrodescendencia local, a partir de las experiencias encarnadas, cuerpos que resisten al olvido, cuidadores de memorias. En otras palabras, la materialidad de la memoria también da cuenta de la experiencia de los antepasados, las cuales se convierten en orientaciones para la agencia de los actuales miembros de la comunidad. Se trata de microrelatos de presencias y ausencias inscriptas en objetos materiales custodiados en la Capilla que desafían el tiempo lineal y homogéneo de la nación (Bhabha 2002[1994]). La materialidad es el sustento de las reivindicaciones: conservar es importante porque ahí viven los antepasados. La presencia física y simbólica de la Capilla de los Negros conlleva un poder de denuncia de las tensiones racializadas de ayer y de hoy, y es signo de una diferencia afroargentina situada en el presente, en contra de la invisibilización y estigmatización que siguen reproduciéndose en diferentes instancias.

Esta experiencia da cuenta que las activaciones de las memorias a través del patrimonio ponen en juego diferentes maneras de representar un pasado traumático de acuerdo a las necesidades del presente, conforme a las luchas por la autoidentificación y las demandas de grupos subalternos (Hill, 1992; Crespo et al. 2007). En este sentido, el recurso a la memoria se establece como estrategia de legitimación a la hora de reconstruir una tradición compartida, movilizando reivindicaciones identitarias de las comunidades y de revisión de un pasado conflictivo (Hoffmann, 2000). Las definiciones derivadas de organismos internacionales sobre patrimonio cultural se basan a partir de la dicotomía material e inmaterial, que recorta y reifica experiencias y memorias de grupos subalternos o porque reproduce saberes globales académicos (Carozzi 2003; Guanche 2004). Particularmente, la noción misma de patrimonio puede concebirse como una categoría antropológica de carácter ambiguo que oscila entre lo material y lo inmaterial, contrastando esta noción con un abordaje propiamente antropológico del patrimonio que de cuenta de los distintos usos, prácticas, experiencias y sentidos sociales en términos etnográficos, teniendo en cuenta el poder como elemento clave en los procesos de patrimonialización (García Canclini 1994, Rotman, 2001; Sampaio Güimaraes, 2016). La separación del patrimonio material del inmaterial como esferas autónomas, no da cuenta de la experiencia misma de los sujetos que "habitan" los espacios físicos y las múltiples manifestaciones culturales y que usan e interpelan esa misma materialidad para fines precisos. La patrimonialización de sitios de memoria en este sentido responde a una objetivación de "otros culturales" y de "experiencias históricas impensables" - la esclavitud al centro de la modernidad, como diría Trouillot, pero a la vez asumida por los sujetos que se basan en la materialidad del sitio físico en cuanto "está ahi" y desde ese lugar disputar reconocimiento y legitimidad, en este caso a través de la RdE de UNESCO.

Nos alejamos entonces de una idea de cultura y de patrimonio cultural como campos inocuos y a-conflictivos, "espacios de encuentros" y de "reconciliación", según lo expresado por los documentos de la RdE UNESCO que citamos en el texto. Por lo contrario, es fundamental tener en cuenta las estructuras sociales del poder que se establecen a partir de las jerarquías hegemónicas étnico/ raciales, de género/sexuales y de clase social, es decir la lógica colonial aplicada a la modernidad (Mignolo, 2010). Las memorias silenciadas y el patrimonio cultural de la diáspora africana en América representan los escenarios de la cultura política y de la política cultural en donde se expresan las dimensiones de la diferencia y del pluralismo histórico (Hall, 2010; Segato, 2007), y desde donde es posible desafiar las lógicas racializadas en el campo del poder y del saber, más allá de las buenas intenciones de los organismos internacionales y de los actores locales.

\section{Referencias bibliográficas}

Alonso, G. F. (Coord.), 2002-2003. La ruta del esclavo. Proyecto archivos Trata de Esclavos. UNESCO, NORAD, Archivo General de la Nación - Memory of de World. 
Alonso, G. F., 2005. Estudio del comercio de esclavos en el Río de la Plata. Archivo General de la Nación Argentina. Memorias del simposio La Ruta del Esclavo en el Río de la Plata: su historia y sus consecuencias, Montevideo: UNESCO, pp. 41-54.

Álvarez Nazareno, C., 2012. El movimiento afro en Argentina en el año del Bicentenario. Pineau, M. (Ed.), Huellas y legados de la esclavitud en las Américas. Proyecto Unesco La Ruta del Esclavo, pp. 173-182, Buenos Aires: UNESCO-EDUNTREF.

Andrews, G. R., 1989. Los Afro-Argentinos de Buenos Aires, Buenos Aires: Ediciones de la Flor.

Annecchiarico, M., 2016. Senderos de la diáspora africana en Argentina y en Cuba. Prácticas y políticas culturales en contexto. Tesis de Doctorado, Facultad de Filosofía y Letras, Universidad de Buenos Aires.

Annecchiarico, M., 2015. Fiesta, memoria y performance en el espacio público. Políticas culturales y reconfiguraciones afro en La Habana y en Buenos Aires. En: C. Crespo, H. Morel y M. Ondelj (Comp.), La política cultural en debate. Diversidad, performance y patrimonio cultural, pp. 51-90. Buenos Aires: Editorial CICCUS.

Annecchiarico, M., 2014. Patrimonio cultural afroargentino: trayectorias, estudios y desafíos. González Noriega, E. (Presidente), Actas de la XII Conferencia internacional antropología, Instituto Cubano de Antropología, La Habana. Del 24 al 29 de noviembre.

Annecchiarico, M., Martin, A. y Mercado, C. 2017. Dimensiones afro en el tango. Tensiones racializadas en los géneros populares del Río de la Plata. Antropologías del Sur, Revista de la Escuela de Antropología, Universidad Academia de Humanismo Cristiano, Año 4, n 5.

Arantes, Antonio A. (org.) 1984. Produzindo o passado. Estrategias de construçao de patrimonio cultural, Brasiliense, Sao Paulo

Bhabha, H. 2002 [1994]. El lugar de la cultura. Buenos Aires: Manantial.

Bilbao, M. A., Cejas, E. y Luayza, N. s/f. Prácticas religiosas de los afrochascomunenses en la Capilla de Los Negros de Chascomús. Informe para UNESCO. Inédito.

Carré, A. M. y Lagrega L. I., 1998. Capilla de los Negros de Chascomús. Algunas cuestiones en torno a su resignificación. Revista de historia bonaerense, Instituto y Archivo Histórico Municipal de Morón, 16: 59-62.

Crespo, C., Losada, F. y Martín, A. (Eds.). 2007. Patrimonio, políticas culturales y participación ciudadana. Buenos Aires: Editorial Antropofagia.

Cruces, F. 1998. Problemas en torno a la restitución del patrimonio. Una visión desde la antropología. Alteridades N. 8 (16). México, pp. 75-84.

Domínguez, M. E., 2009. 'Suena el río'. Milongas, candombes, murga y tango en los músicos rioplatenses. Tesis de Doctorado. PPGAS, Universidad Federal de Santa Catarina.

Fernández Bravo, N. 2012. Qué ha cambiado en el 'campo afro' de la Argentina? Temporalidades, paradojas, desafíos. En Annecchiarico, M. y Martín, A. (Eds), Afropolíticas en América del Sur y el Caribe, pp.73-88. Buenos Aires: Ediciones Puentes del Sur.

Ferreira, L., 2008. Música, artes performáticas y el campo de las relaciones raciales. En: G. Lechini (Comp.). Los estudios afroamericanos y africanos en América Latina. Herencia, presencia y visiones del otro, pp. 225-250. Córdoba: CLACSO-CEA/UNC.
Ferreira, L., 2009. Demandas de los movimientos sociales afrolatinoamericanos en materia de diversidad cultural en el proceso de Durban (III Conferencia Mundial Contra el Racismo) y de su seguimiento. En: Actas de la VIII RAM - Reunión de Antropología del Mercosur, Buenos Aires.

Frigerio, A., 2008. De la desaparición de los negros a la reaparición de los afrodescendientes: comprendiendo las políticas de las identidades negras, las clasificaciones raciales y de su estudio en Argentina. Buenos Aires: CLACSO, C.E.A.- Universidad Nacional de Córdoba.

Frigerio, A. y Lamborghini, E., 2011. (De)mostrando cultura: estrategias políticas y culturales de visibilización y reivindicación en el movimiento afroargentino. Boletín Americanista, Barcelona, Año XI 2, 63: 101-120.

García Canclini, N. 1994. ¿Quiénes usan el patrimonio? Políticas culturales y participación social. Memorias del Simposio Patrimonio y Política cultural para el siglo XXI. México: INAH.

Geler, L., 2016. Categorías raciales en Buenos Aires. Negritud, blanquitud, afrodescendencia y mestizaje en la blanca ciudad capital. Runa. Archivo para las ciencias del hombre, Buenos Aires, 37(1), 71-87.

Geler, L., 2010. Andares negros, caminos blancos. Afroporteños, Estado y Nación Argentina a fines del siglo XIX. Rosario: Ediciones Prehistoria.

Gilroy, P., 2003 [1993]. The Black Atlantic. L'identità nera tra modernità e doppia coscienza. Roma: Meltemi.

Gomes, M., 2011. La diáspora africana en la Argentina. Pineau, Marisa (Ed.). La Ruta del Esclavo en el Río de la Plata. Aportes para el diálogo intercultural, pp. 431-438. Buenos Aires: UNESCOEDUNTREF.

Hall, S., 2010. Sin garantías. Trayectorias y problemáticas en estudios culturales. E. Restrepo, Catherine Walsh y Víctor Vich (Eds.), Popayán: Envión Editores.

Halperin, F., 2003. Reconstruyen la ruta de los esclavos en la época colonial. Diario La Nación, 17 de mayo de 2003.

Hill, J. D., 1992. Contested Pasts and the Practice of Anthropology. American Anthropologist, vol. 94, 4: 809-815.

Hoffmann, O., 2000. La movilización identitaria y el recurso a la memoria. (Nariño, Pacífico colombiano). C. Gnecco y M. Zambrano (Eds). Memorias hegemónicas, memorias disidentes, pp. 97-120Cauca: ICAN - Universidad del Cauca.

Informe de la Conferencia Mundial contra el Racismo, la Discriminación Racial,la Xenofobia y las Formas Conexas de Intolerancia, Durban, 31 de agosto a 8 de septiembre de 2001, A/CONF.189/12.

Lahourcade, A., 1973. La comunidad negra de Chascomús y su reliquia, Chascomús: s/e.

Lahourcade, A., 2008. Mural de la negritud. Buenos Aires: Ed. Dunken.

Lao-Montes, A., 2007. Decolonial moves: Trans-locating African diaspora spaces. Cultural Studies, Vol. 21, (2-3):309-338.

López, L., 2006. Organización política y articulación con espacios locales-globales de los afrodescendientes en Argentina en la última década. L. Maronese (Comp.). Buenos Aires negra. Identidad y cultura, Temas de Patrimonio Cultural, Buenos Aires, 16: 99-114. 
López, L. 2012. Que América Latina se sincere: los movimientos negros frente a los actuales desafíos de políticas públicas y reparaciones en el Cono Sur. M. Annecchiarico y A. Martín (Eds.), Afropolíticas en América Latina y el Caribe, pp. 99-119. Buenos Aires: Editorial Puentes del Sur.

Luongo, M. T., 2013. Argentina Rediscovers Its African Roots. The New York Times. 12/09/2013. Recuperado de: www.nytimes. com/2014/09/14/travel/argentina-rediscovers-its-african-roots. html?_r=0 (Consultado el 15 de junio de 2017).

Martín, A., 2015. Candombe, progreso y blanqueamiento forzado en los festejos del carnaval de Buenos Aires a fines del siglo XIX. C. Carolina, H. Morel y M. Ondelj (Comp.). La política cultural en debate. Diversidad, performance y patrimonio cultural, pp. 21-50. Buenos Aires: Editorial CICCUS.

Meeting of the Scientific Committee of the UNESCO Slave Route Project : Resistance, Liberty and Heritage, 2012. Calabar, Nigeria. Documento digital.

Mignolo, W. 2010. Desobediencia epistémica: retórica de la modernidad, lógica de la colonialidad y gramática de la descolonialidad. Buenos Aires: Ediciones del Signo.

Monkevicius, P. C., 2012. "No tenía que haber negros": memorias subalternas y visibilización entre afrodescendientes e inmigrantes africanos en Argentina. Publicar, año X n XII: 87-105.

Morel, H. 2001. 'Milonga que va borrando fronteras'. Las políticas del patrimonio: un análisis del tango y su declaración como Patrimonio Cultural Inmaterial de la Humanidad. Revista Intersecciones en Antropología, 12, Olavarría.

Mosquera, C. y Barcelos, L. C. (Eds), 2006. Afro-reparaciones: memorias de la esclavitud y justicia reparativa para negros, afrocolombianos y raizales. Bogotá: Universidad Nacional de Colombia.

Ochoa, A. M., 2002. Políticas culturales, academia y sociedad. En Daniel Mato (Coord) Estudios y otras prácticas intelectuales latinoamericanas en cultura y poder, pp. 213-224. Consejo Latinoamericano de Ciencias Sociales, CEAP, FACES, Universidad Central de Venezuela, Caracas.

Pacheco, R. 2008. Bibliografía afro-porteña (1999-2003): invisible, pero no olvidada. Goldman, G. (Comp.), Cultura y sociedad afrorioplatense, Montevideo: Perro Andaluz Ediciones.

Parody, Viviana. 2013. 'Tambores de Plaza Dorrego': performance, territorio y memoria en la práctica 'tradicional' de candombe afrouruguayo en Buenos Aires". Ghidoli, M. L. y Martínez Peria, J. F. (Comps.), Estudios Afrolatinoamericanos. Nuevos enfoques multidisciplinarios. Actas de las Terceras Jornadas del GEALA, Ediciones del Centro Cultural de la Cooperación Floreal Gorini.

Picotti, D. (Comp.), 2001. El negro en la Argentina: presencia y negación, Buenos Aires. Editores de América Latina.

Picotti, D., 2012. Capilla de los Negros. En UNESCO, Sitios de memoria y culturas vivas de los afrodescendientes. Argentina, Paraguay y Uruguay. Tomo I. Huellas e Identidades, Montevideo: UNESCO, pp.12-29.

Pineau, M. (Ed.), 2012. Huellasylegados de la esclavitud en las Américas. Proyecto Unesco La Ruta del Esclavo, Buenos Aires: UNESCO-EDUNTREF.

Pineau, M. (Ed.), 2011. La Ruta del Esclavo en el Río de la Plata. Aportes para el diálogo intercultural. Buenos Aires: UNESCOEDUNTREF.
Pineau, M. y Efron, L. 2009. Seminario La ruta del esclavo en el Río de la Plata: Aportes para un diálogo intercultural. Estudios Históricos - CDHRP, n. 3.

Pita, F., 2012. El pasado es tan inquietante como el presente y tan incierto como el futuro. En: Pineau, M. (Ed.), Huellas y legados de la esclavitud en las Américas. Proyecto Unesco La Ruta del Esclavo, pp. 171-172. Buenos Aires: UNESCO-EDUNTREF.

Pollak, M., 2006. Memoria, olvido, silencio. La producción social de identidades frente a situaciones límite, La Plata: Ediciones Al Margen.

Prats, L. 1997. Antropología y Patrimonio, Barcelona, Ed. Ariel.

Rotman, M., 2001. Legitimación y preservación patrimonial: la problemática de las manifestaciones culturales 'no consagradas'. Temas de Patrimonio. Memorias, Identidades e Imaginarios sociales. Comisión para la preservación del patrimonio histórico cultural de la ciudad de Buenos Aires, n.5, pp. 154-168

Sampaio Güimaraes, R. (2016). Entre memorias de Portugal y de África: las políticas patrimoniales en la región portuaria de Río de Janeiro, Brasil. Quadernos de Antropología Social, n. 44: 51-66

Schávelzon, D., 2003. Buenos Aires negra. Arqueología histórica de una ciudad silenciada, Buenos Aires: Emecé Editores.

Segato, R. L., 2007. La nación y sus otros: raza, etnicidad y diversidad religiosa en tiempos de políticas de la identidad. Buenos Aires: Prometeo Libros.

Trouillot, R-M., 1995. Silencing the Past. Power and the production of History. Boston: Beacon Press.

UNESCO, 1993. 'Memory of te World' Programme. Suggested guidelines for the protection of endangered manuscripts and archives. Arnoult, Jean-Marie (Ed). París, UNESCO. http://unesdoc. unesco.org/images/0009/000962/096263eb.pdf (consultado el 15 de junio de 2018).

UNESCO, 2009. La Ruta del esclavo. Reconciliar el deber de memoria con la verdad histórica. París: UNESCO. http://unesdoc.unesco.org/ images/0018/001866/186636s.pdf (consultado el 15 de junio de 2018).

UNESCO, 2005. La Ruta del Esclavo en el Río de la Plata: su historia y sus consecuencias. Montevideo: UNESCO. http://unesdoc.unesco. org/images/0015/001509/150922s.pdf (consultado el 15 de junio de 2018).

UNESCO, 2006. Nueva estrategia para el Proyecto Ruta del Esclavo. París: UNESCO.

UNESCO, 2012. Sitios de Memoria y culturas vivas de los afrodescendientes en Argentina, Paraguay y Uruguay. Tomo I Huellas e identidades. Montevideo: UNESCO. http://unesdoc.unesco.org/ images/0022/002277/227700s.pdf (consultado el 15 de junio de 2018).

UNESCO, 2014. La Ruta del Esclavo: 1994-2014. El camino recorrido. París: UNESCO. http://unesdoc.unesco.org/ images/0022/002284/228475s.pdf (consultado el 15 de junio de 2018).

Velázquez Gutiérrez, M. E. 2012. Carta dirigida a Irina Bokova, Directora General de UNESCO. Ciudad de México. Documento digital.

Wade, P., 2006. Etnicidad, multiculturalismo y políticas sociales en Latinoamérica: Poblaciones afrolatinas (e indígenas). Tabula Rasa, $n^{\circ} 4,: 59-81$. 